\title{
Editorial: OJPHI Vol 4, No 3 (2012)
}

Welcome to the last issue of the 4th volume of the Online Journal of Public Health Informatics. I am sure you are all aware that the National Library of Medicine has approved our application for indexing and archiving in PubMed Central. The next step is to deliver all past issues of the journal to NLM in the required XML DTD format. The NLM will provide links to other related biomedical informatics journals. This process will be completed in February 2013. We can all proudly say that we now have a scholarly biomedical informatics journal dedicated to the exciting field of public health informatics.

This issue contains nine original articles, two review articles, a technology review, and a commentary. The topics covered in this issue include the identification of some fundamental concepts and factors that must be understood if public heath informatics applications are to support high-level cognitive activities; the development of computerized decision support systems to capture structured clinical data from providers using office-based fax machines and the delivery of just-in-time alerts to PCPs statewide; identification of information requirements and barriers to information exchange by public health workers; development of a methodology to establish and maintain successful electronic health record exchanges between clinical practices and public health agencies; the use of data from the Behavioral Risk Factor Surveillance Systems surveys to study dental health disparities; the development of an develop an agent-based model to simulate the spread of sexually transmitted infections in a population; the use of institutional logics perspective to analyze the implementation of health information systems in a developing county; the development and use a modified Health Belief Model to improve the predictive power of factors that influence healthy eating behavior; an analysis of the history, issues, and potentials for successful implementation of electronic prescribing in the current health reform environment; improvement of HIV/AIDS knowledge management using electronic health records; the development of a methodology to facilitate communication between open source and propriety systems using interoperability principles; and a technology review on leveraging cloud computing to develop Smarter Public Health Prevention Systems (SPHS) to provide real-time reports of potential public health threats; and a commentary on identification of successes and challenges in the use of mobile phone applications in health and demographic surveillance systems in a developing country.

It has long been noted by researchers that human and non-technical factors, rather than technological defects, contribute significantly to failures in implementation of public health informatics (PHI) applications. In order to improve the success rate of PHI tools it is important to pay more attention to human-centric design issues rather than completely focusing on technology issues. In the paper titled "Beyond information access: Support for Complex Cognitive Activities in Public Health Informatics Tools" the authors draw on research from the general area of human-information interaction in complex cognitive activities to identify some of the extant research needs of public health informatics tools. They also discuss a number of considerations for the design and evaluation of PHI tools, and demonstrate how an integration of such considerations facilitates the design and evaluation of successful PHI tools. In order to improve the successful adoption of electronic health records and health information exchanges by health 
departments in the current HITECH era it is important to encourage the participation of public health professionals in the design discourse.

The secure sharing of protected health information is a fundamental determinant of the success of the HITECH Ac. An insignificant percentage of hospitals currently share structured data via the health information exchanges. The participation of majority of practices in the HIEs consists of using the office-based faxes to receive results or to log onto the HIE portal, where one exists, to receive health information. In the paper titled "Real Time Alert System: A Disease Management System Leveraging Health Information Exchange", the authors developed a computerized decision support system (CDSS) using existing HIE infrastructure. The CDSS captures structured clinical data from providers using existing office-based fax machines and delivers real time alerts for recommended services to PCPs in the statewide Indiana Network for Patient Care when their patients visit. The technology was evaluated for emergency room visits anywhere in the state of Indiana. The results showed that the CDSS successfully delivered the just-in-time alerts to the PCPs across the state. Physicians who reported finding the information helpful also reported making a follow-up phone call or seeing the patient for a follow-up care. This study demonstrates that, even before the implementation of health information exchanges on a national level, most states can use such a CDSS to coordinate care, improve outcomes, and reduce costs of care.

An understanding of the information requirements and barriers to information exchange by public health workers should precede information systems design efforts. In the paper titled "Public Health Practice within a Health Information Exchange: Information Needs and Barriers to Disease Surveillance", Reeder, Revere, Hills, et.al, investigated information usage by public health professionals working on disease surveillance activities at a medium-sized health department. Their results indicated barriers in information systems usability; data timeliness, accuracy, and completeness; and social interaction with clients. In orders to improve the adoption of information systems and implementation of information exchanges by public health departments systems designers must address these barriers.

In order for clinicians to make the best possible clinical and economically responsible vaccination decisions it is important to have access to information about the earliest possible intervals that are safe to administer vaccinations, especially to children at highest risk of vaccine preventable diseases. The required data are contained in state Immunization Information Systems (IIS) and registries. The Meaningful Use Stage 1 standards require providers and hospital EHRs to demonstrate the ability to send immunization data to an IIS while the Meaningful Use Stage 2 standards include the requirement to send clinically correct and complete immunization records from the provider's EHR to an IIS. In a paper titled "A ThreeStep Approach for Creating Successful Electronic Immunization Record Exchanges between Clinical Practice and Public Health", Janet Balog presents a methodology to establish and maintain electronic health record exchanges; demonstrates the value of clinical and technical testing before implementation of data exchanges; and discusses how the Meaningful Use requirement to advance data exchange with public health agencies achieves mutual health outcomes for providers and public health programs.

Monitoring health disparities is a major activity of the Healthy People 2020 initiative. Research shows that the most important determinants of oral health are poverty, race and ethnicity. In the paper titled "Overcoming Data Challenges Examining Oral Health Disparities in Appalachia", 
the authors use the Behavioral Risk Factor Surveillance Systems survey data to study dental health disparities. Using Beale codes to define metropolitan and non-metropolitan statistical areas and GIS maps the authors provide informative sub-state results to assist health planners in targeting oral health intervention strategies.

While public health professionals devote significant amount of time and money to screen and treat STISs annually this cluster of diseases remain a major public health challenge. Estimates from CDC show that approximately 19 million new cases of STIs occur in the U.S. each year, costing the healthcare system $\$ 12$ billion to $\$ 20$ billion annually. In recent decades researchers are increasingly employing agent-based models to simulate the spread of sexually transmitted infectious diseases as a complementary approach to the traditional statistical or differential equation-based models. In the paper titled "An Agent-Based Model for Simulating the Spread of Sexually Transmitted Infections", Rutherford, Friesen, et.al, develop an agent-based model to simulate the spread of sexually transmitted infections in a population of 1000 agents over a 10year period. The model allows the effects of various mitigating and control policies and behaviors to be analyzed. The results show that changes in individual behaviors can reduce the risk of exposure. However, population-wide behavior modifications through public health activities have can have more dramatic impacts on the transmission of STIs.

The healthcare sector is one of the most information-intensive enterprises and health information systems (HIS) are important components of health reform. Well-developed and implemented health information systems can improve the coordination of care, reduce duplications and errors, improve access, quality, and reduce costs. However, even in the U.S., less than 50 percent of health information systems are outright successes, delivering the expected functionalities on time and within budget. This significant failure has been attributed to the complexities of the technologies and the difficulty in satisfying the multiple conflicting objectives of the many stakeholders involved. The situation is even worse in developing countries. In a paper titled "Understanding HMIS Implementation in a Developing Country Health Country" Ime Asangansi employs the institutional logics perspective to analyze HMIS implementation in a state government ministry in Northern Nigeria. The author recognizes that certain institutional logics may be conflicting and lead to increase in the risk of failure. The following institutional logics were identified: a) the logic that sustainability can only be achieved through local ownership or the local control logic, b) the logic of universal coverage, and c) the logic of network-centric organizations where network technologies disrupt institutional power structures. It is proposed that the resolution of the conflicting logics must be addressed within the context of deinstitutionalization, changeover management, and by balancing the competing interests. A major recommendation from the study is that, in order to improve the implementation of HMIS in developing countries, policy makers must understand and resolve the conflicting logics

The increase in lifestyle-related health problems and Meaningful Use requirements provide incentives to providers to shift their practices from the current treatment-and-prescription centric models to patient-and population centric prevention and health promotion models. When Meaningful Use Stage 2 and Stage 3 requirements are enforced in future healthcare providers will be expected to provide patients with information that will help them make lifestyle changes. Health application and game designers are already developing behavior modification programs to assist patients in making healthy choices. Behavior modification models based on intuition are 
less successful or sustainable than those informed by evidence-based research. One of the most widely used theories for behavior modification is the Health Belief Model (HBM) which was developed to investigate why people fail to undertake preventive health measures. The main limitation of this model is that it has very low predictive power. In a paper titled "Towards an Effective Health Intervention Design: An Extension of the Health Belief Model", the authors extended the HBM to include new lifestyle changing factors and tested the validity of the extended HBM and the original model on healthy eating behavior. The results showed a significant increase in the predictive power of the extended model over the original model. As the health information exchanges mature and generate individual and population level databases researchers will use these data to create new evidence for lifestyle modifications in order to achieve the objectives of the Accountable Care Act.

This is the final issue of the Online Journal of Public Health Informatics in 2012.Many thanks to the editors, the journal manager, and all the volunteers who have contributed to the success of this project. Through your hard work the field of public health informatics can now boast of a scholarly journal dedicated to the dissemination of evidence-based information to our stakeholders. I look forward to your continued support in 2013 and beyond.

Happy New Year!

Edward Mensah, $\mathrm{PhD}$

Editor-in-Chief

Online Journal of Public Health Informatics

1603 West Taylor Street, Room 759

Chicago, Illinois, 60612

Email:dehasnem@uic.edu

Office: (312) 996-3001 